\title{
Design of a 60 kA Flux Pump for Fusion Toroidal Field Coils
}

\author{
James H.P. Rice Student Member, IEEE, Jianzhao Geng, Chris W. Bumby, Hubertus W. Weijers Senior \\ Member, IEEE, Steven Wray, Heng Zhang, Frank Schoofs and Rodney A. Badcock Senior Member, IEEE.
}

\begin{abstract}
High-temperature superconducting (HTS) magnets offer a novel pathway to magnetically confined fusion energy generation. Reactor-scale fusion devices require tens of $\mathrm{kA}$ of current to generate large toroidal fields for magnetic confinement. Operating HTS magnets at kA-current levels is difficult due to unavoidable joint resistances that require active driving of current. The large footprint and energy demand of a solidstate power supply and multi-kA current leads present significant constraints on the size, cost and efficiency of a fusion tokamak. An alternative approach is to use an HTS flux pump, which can generate and sustain persistent currents in HTS coils at the kAlevels required by fusion magnet systems. Here, we outline the design of a $60 \mathrm{kA}$ transformer-rectifier flux pump for energizing and sustaining an $80 \mathrm{mH}$ HTS fusion magnet. The flux pump is driven by an iron-core transformer with copper primary and HTS secondary windings. This is rectified by switching elements in the superconducting circuit into a uni-directional dc voltage output which charges the inductive load. To charge the load coil to $60 \mathrm{kA}$ in 24 hours, an output dc voltage of $55 \mathrm{mV}$ is needed. However, only $6 \mathrm{mV}$ is required to maintain a long-term steady current of $60 \mathrm{kA}$. Such a device can significantly reduce the cost and footprint of future fusion reactor designs.
\end{abstract}

Index Terms-Current Leads, Fusion Energy, HTS Flux Pump, Tokamak, Transformer-Rectifier.

\section{INTRODUCTION}

$\mathbf{F}$ USION energy aims to produce a large amount of electrical power to national grids in a way that is safe, sustainable, and economic. A prominent method of magnetic confinement is the tokamak, which uses strong magnetic fields from toroidal field (TF) coils to confine the fuel plasma. Generating the high fields necessary uses large, superconducting electromagnets [1], [2]. The ITER project [3] in France gives a good indication of a reactor-scale tokamak. ITER's magnet systems contain $18 \mathrm{Nb}_{3} \mathrm{Sn} \mathrm{TF}$ coils, each carrying $68 \mathrm{kA}$ to generate a toroidal field of $5.3 \mathrm{~T}$, with a total stored energy of 41 GJ. This corresponds to an inductance of roughly $250 \mathrm{mH}$ per magnet coil. Energizing and sustaining such an inductive load at high current is extraordinarily difficult, requiring large, reactive power-electronic systems [4]. The superconducting

This work was supported in part by the New Zealand Ministry of Business, Innovation and Employment (MBIE RTVU1916), and has been part-funded by STEP, a UKAEA programme to design and build a prototype fusion energy plant and a path to commercial fusion.

J.H.P. Rice, J. Geng, C. W. Bumby, H. W. Weijers, and R.A. Badcock are with the Robinson Research Institute, Victoria University of Wellington, Lower Hutt 5010, New Zealand. (e-mail: james.rice@ vuw.ac.nz).

J. Geng is also with the Huazhong University of Science and Technology, Wuhan, Hubei, China.

S. Wray, H. Zhang and F. Schoofs are with the UK Atomic Energy Authority, Culham Science Centre, Abingdon OX14 3DB United Kingdom.

Manuscript received September 6th, 2021. magnets operate at cryogenic temperatures, and current must be conveyed into the cryostat using current leads [5]. Current leads inevitably leak heat into the cryostat; the amount of which depends on the current lead design [6]. Such heat must be removed at penalty, making it costly [7]. Moreover, current is generated using resistive components, whose loss goes as $I^{2} R$, further increasing the required power input. Overall, these factors reduce the engineering power gain $Q_{E}$ of the reactor, and negatively impact the economic viability of the reactor.

Alternatively, high-temperature superconducting (HTS) flux pumps are superconducting power supplies [8]-[18] that can energize high currents in inductive superconducting load coils. Load current outputs of $>1 \mathrm{kA}$ have been demonstrated in recent years [19]-[21]. Current can then be sustained indefinitely in a quasi-persistent current mode [22], whereby the flux pump actively replaces the energy lost to joint resistances in series with the magnet. This gives them significantly lower internal impedance than conventional supplies [23]. HTS flux pumps wirelessly generate voltage within the cryostat itself [24], foregoing the need for current leads to room temperature and their associated losses. Utilizing flux pumps to power tokamak TF coils may significantly reduce required input power, improving the overall power balance of tokamak reactors.

Transformer-rectifier flux pumps (TRFP) [13]-[15] act as ac current to dc voltage converters. Ac current is induced in a superconducting circuit and rectified into dc voltage across a load coil by using superconducting switching elements. The circuit can be arranged in half- or full-wave configurations, as shown in Fig. 1. In either case, the flux pump can be closely modelled using a simple circuit topology [25]. The transformer wirelessly transfers ac current from a room temperature primary to a superconducting secondary. The ac waveform is shown in Fig. 1c). Switching elements can employ either dynamic resistance from ac loss [13], [23] or non-linear resistance [14], [26], [27] to generate voltage. The load current is sustained between charging cycles through the parallel 'bridge' switch which in the 'closed' state has no resistance. To prevent the transformer from saturating [28], flux across the switches must be conserved within a cycle to avoid the build up of trapped flux in the charging loop [21]. Flux motion across the switching element results in a circuit voltage, allowing this requirement to be stated using the cycleaveraged integral of voltage across the series and parallel switches as

$$
\frac{1}{T} \int_{0}^{T} v_{1} \mathrm{~d} t=\frac{1}{T} \int_{0}^{T} v_{2} \mathrm{~d} t
$$



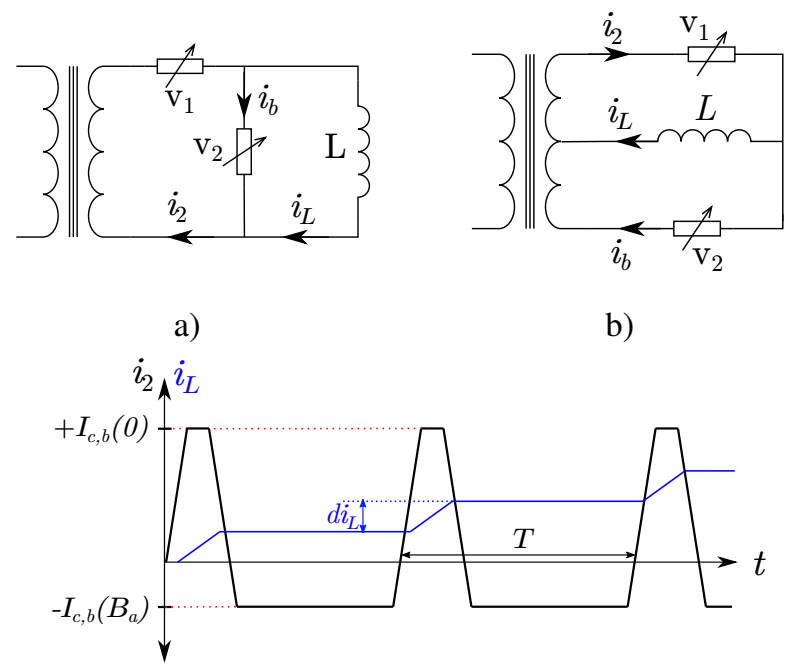

c)

Fig. 1. Circuit diagrams of both a) half- and b) centre-tap full-wave TRFP. Switches are depicted as variable resistors, $v_{1}$ and $v_{2}$, and the load coil as the inductor $L$. The transformer is taken to have a resistive copper primary and superconducting secondary. c) Input trapezoidal waveform with transformer secondary current $i_{2}$ (black) and load current $i_{L}$ (blue), with load current charging increment $d i_{L}$.

where $f=1 / T$ is the frequency of the input current, and $v_{1}$ and $v_{2}$ are the instantaneous voltages across the series and parallel switches, respectively.

Until now, little attention has been given to the power efficiency of HTS flux pumps [27], [29]. Power transfer efficiency can be used to relate output power to the heat loss of switching, and therefore the losses in the cryostat, making it a useful tool in calculating the expected cryogenic heat load of a flux pump.

\section{IdEAL FLuX PumP EFFICIENCY}

The efficiency $\eta$ is the ratio of output power delivered to the load and total power dissipated in the circuit.

$$
\eta=\frac{P_{\text {out }}}{P_{\text {out }}+P_{\text {sw }}}
$$

where $P_{\text {out }}$ is the output power to the load, and $P_{\mathrm{sw}}$ is the power dissipated in switching components. Beginning with the half-wave rectifier (Fig. 1a)), these powers are given as

$$
\begin{gathered}
P_{\text {out }}=\frac{1}{T} \int_{0}^{T} v_{2} \cdot i_{L} \mathrm{~d} t, \\
P_{\mathrm{SW}}=\frac{1}{T} \int_{0}^{T}\left(v_{1} \cdot i_{2}+v_{2} \cdot i_{b}\right) \mathrm{d} t,
\end{gathered}
$$

where $i_{2}, i_{b}$, and $i_{L}$ are the instantaneous transformer secondary, bridge, and load currents, respectively. Instantaneous values of current and voltage are distinguished from steadystate values by the use of lower case. Substituting into (2) leads to

$$
\eta=\frac{\int_{0}^{T} i_{L} \cdot v_{2} \mathrm{~d} t}{\int_{0}^{T} i_{L} \cdot v_{2} \mathrm{~d} t+\int_{0}^{T}\left(v_{1} \cdot i_{2}+v_{2} \cdot i_{b}\right) \mathrm{d} t} .
$$

Eq. (5) can be used to numerically calculate ideal efficiency each cycle.

Rectification occurs when the cycle-average of bridge voltage $v_{2}$ is non-zero, resulting in net dc voltage across the load. For sufficiently small injections of current in each cycle, for example at load saturation with low current ripple, $d i_{L} \ll i_{L}$ and $i_{L}$ can be removed from the integral as a constant.

$$
\eta=\frac{i_{L} \int_{0}^{T} v_{2} \mathrm{~d} t}{i_{L} \int_{0}^{T} v_{2} \mathrm{~d} t+\int_{0}^{T}\left(v_{1} \cdot i_{2}+v_{2} \cdot i_{b}\right) \mathrm{d} t}
$$

Eq. (1) equates cycle-averaged switching voltages, giving

$$
\eta=\frac{i_{L} \int_{0}^{T} v_{2} \mathrm{~d} t}{i_{L} \int_{0}^{T} v_{2} \mathrm{~d} t+2 \int_{0}^{T} v_{2} \cdot\left(i_{2}+i_{b}\right) \mathrm{d} t} .
$$

Voltage is generated when $I_{t} \geq I_{c}$. Using Bean's critical state model [30], instantaneous currents are limited to critical currents when switched, at which an arbitrary amount of voltage can be produced. This applicability of this assumption will be tested later. This allows current to be removed as constant

$$
\eta=\frac{i_{L} \int_{0}^{T} v_{2} \mathrm{~d} t}{i_{L} \int_{0}^{T} v_{2} \mathrm{~d} t+2\left(I_{2-}+I_{c, b}\left(B_{a, \perp}\right)\right) \int_{0}^{T} v_{2} \mathrm{~d} t},
$$

where $I_{2-}$ and $I_{c, b}\left(B_{a, \perp}\right)$ are the secondary current in the negative part of the ac waveform and bridge critical current under perpendicular applied field, respectively. The resulting efficiency is then independent of voltage waveform.

$$
\eta=\frac{i_{L}}{i_{L}+I_{c, b}\left(B_{a, \perp}\right)+I_{2-}} .
$$

Assuming the two switches are identical, the negative waveform trough cannot exceed $I_{c, b}\left(B_{a, \perp}\right)$.

$$
\eta=\frac{i_{L}}{i_{L}+2 I_{b, c}\left(B_{a, \perp}\right)}
$$

The secondary must supply a positive waveform peak of $i_{2+}=i_{L}+i_{b}$ such that the bridge current exceeds $I_{c}$ to produce voltage when switched. Additionally, to prevent the load current being limited by the bridge when the secondary current is negative, the switches must have a larger self-field critical current $I_{c, b}(0)$ than the combined load and bridge currents.

$$
I_{c, b}(0) \geq i_{L}+I_{2-}=i_{L}+I_{c, b}\left(B_{a, \perp}\right)
$$

Substituting (11) into (10) gives a limiting expression of only critical currents,

$$
\eta \leq \frac{I_{c, b}(0)-I_{c, b}\left(B_{a, \perp}\right)}{I_{c, b}(0)+I_{c, b}\left(B_{a, \perp}\right)} .
$$

This may be simplified by defining a "switching factor" to represent the performance of the switching element

$$
\kappa=\frac{I_{c, b}(0)}{I_{c, b}\left(B_{a, \perp}\right)},
$$

which gives efficiency as

$$
\eta \leq \frac{\kappa-1}{\kappa+1} .
$$




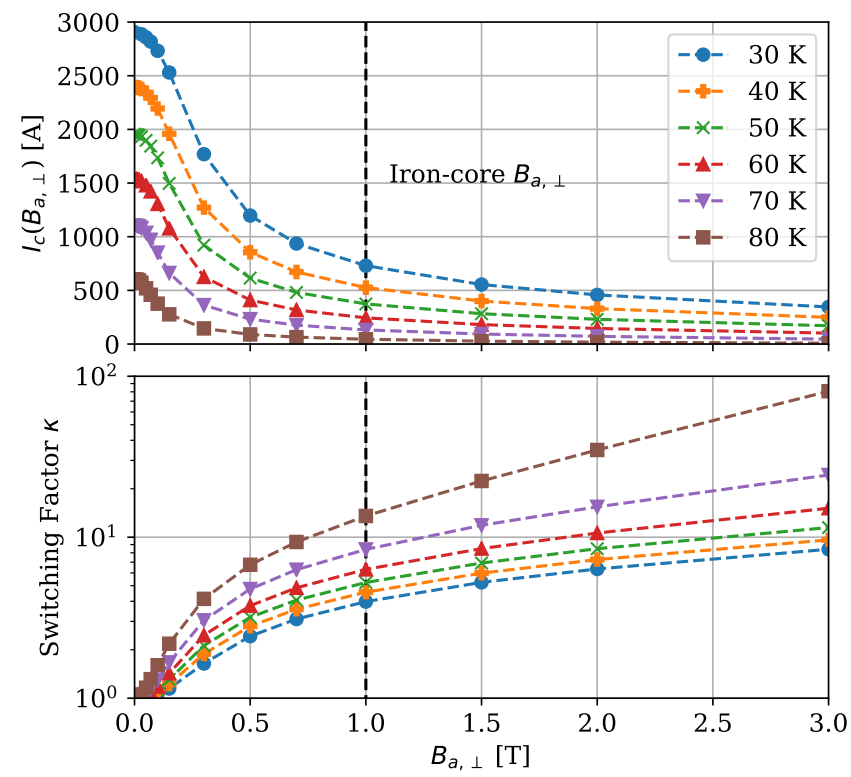

Fig. 2. $I_{C}(B)$ response of SCN12700-210222-01 HTS tape, manufactured by SuNAM Co. Ltd., and resulting switching factor $\kappa$ at different temperatures. HTS tape was commercially characterized at the SuperCurrent facility [31], [32]. An assumed iron-core $B_{a, \perp}$ of $1.0 \mathrm{~T}$ is shown as a black-dashed line.

This analysis can be extended to the center-tap full-wave configuration (Fig. 1b)) by noting that similar limiting conditions on critical current (11) and flux conservation (1) apply. The input waveform need only be asymmetric for a halfwave or passive rectifier; full-wave uses a symmetric $(50 \%$ duty) square-wave of $\pm I_{c, b}\left(B_{a, \perp}\right)$ [10], which will reduce the requirements of the input transformer.

In the period of a cycle, the power delivered to the load in a centre-tap full-wave configuration is twice that of a half-wave:

$$
\begin{gathered}
\eta=\frac{2 i_{L}}{2 i_{L}+2 I_{c, b}\left(B_{a, \perp}\right)} \\
\eta \leq \frac{I_{c, b}(0)-I_{c, b}\left(B_{a, \perp}\right)}{I_{c, b}(0)}=\frac{\kappa-1}{\kappa}
\end{gathered}
$$

This shows increased efficiency in the full-wave circuit.

Equations (14) and (16) for the ideal rectifier efficiency are both independent of voltage terms. Therefore, the voltage source and waveform are both considered irrelevant in the ideal case. For a given current output capacity, the self-field critical current is fixed by (11) and the efficiency improves by suppression of critical current under applied field, defined as the switching factor $\kappa$. Increasing $\kappa$ improves the ideal efficiency of the transformer-rectifier circuit in both half- and full-wave topologies.

From (13), $\kappa$ is dependent only on the $J_{c}(B)$ properties of the superconducting tape used in the switch bridge. To interrogate the efficiency directly, the $J_{c}(B)$-characteristics of a candidate Gd-BCO tape, manufactured by SuNAM Co. Ltd., were measured. Characterization was performed at the SuperCurrent Facility at Robinson Research Institute [31], [32] for a range of temperatures and applied fields $B_{a, \perp}$ between $30 \mathrm{~K}$ and $80 \mathrm{~K}$ and up to $3 \mathrm{~T}$, respectively. The results agree with manufacturer specifications at $77 \mathrm{~K}$ and are shown in

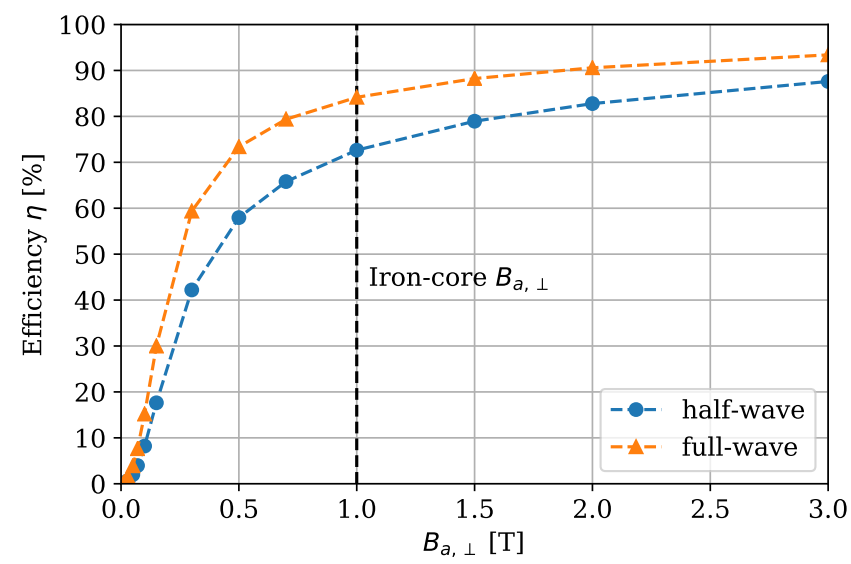

Fig. 3. Ideal Efficiency $\eta$ of both half- (blue) and full-wave (orange) TRFP operating at $60 \mathrm{~K}$ using a range of applied fields $B_{a, \perp}$. Values were calculated from the $60 \mathrm{~K} I_{c, b}\left(B_{a, \perp}\right)$ data in Fig 2 .

Fig. 2. The resulting switching factor $\kappa$ was then calculated as the self-field $\left(B_{a, \perp}=0.0 \mathrm{~T}\right) I_{c}$ divided by $I_{c, b}\left(B_{a, \perp}\right)$.

Expected ideal efficiency was calculated using (14) and (16) with the $60 \mathrm{~K} J_{c}(B)$ data, and the resulting half- and full-wave efficiencies are shown in Fig. 3. In both circuit topologies, efficiency increases at higher applied field due to the improvement in switching factor. Efficiency improves rapidly at lower applied fields, with diminishing returns as $B_{a, \perp}$ increases due to the $J_{c}(B)$ response shown in Fig. 2 . Switching also improves at higher temperature due to the increased $J_{c}(B)$ dependence of the tape close to the critical temperature $T_{c}=91 \mathrm{~K}$. Thus, operating the flux pump at increased temperatures and applied fields gives improved efficiency.

The presented model is deliberately simplistic; neglecting ac and transformer losses, joint resistance, and circuit inductances and use of Bean's model to provide tractability. The use of Bean's model is appropriate for superconductors with large $n$-values $(n \sim 100)$ and imposes that current cannot exceed $I_{c}$, but must be relaxed for superconductors with finite $n$ values such as $2 \mathrm{G}$ HTS wire $(n \sim 20)$. Voltage outputs significantly larger than $1.0 \mu \mathrm{V} / \mathrm{cm}$ would cause a switch made of HTS wire to carry current greater than $I_{c}$, making the ideal assumption that current is limited to only $I_{c, b}\left(B_{a, \perp}\right)$ inappropriate. For example, we will later see that $55 \mathrm{mV}$ is required to charge an example magnet, implying that a $30 \mathrm{~mm}$ section of superconductor must carry $1.6 I_{c}$ to generate such voltage. Future work must relax this assumption to accurately include voltage output in the calculated efficiency. Ac loss terms relating to the changing applied field during switch actuation or the use of dynamic resistance switching will introduce additional magnetization loss. This will not significantly affect the power transfer efficiency during low frequency operation $(<1 \mathrm{~Hz})$ but represents an additional heat loss term. Such ac loss has been effectively modeled in the case of a HTS dynamo by Ghabelli et al. [33], and may become significant at higher operational frequencies. It is also assumed that the transformer is ideal, which is appropriate as long as the frequency of operation is low and the transformer 
TABLE I

DESign CONSTRAints OF REACTOR-LIKE* MAGNET COIL

\begin{tabular}{ll}
\hline \hline Operational Current $I_{\text {coil }}$ & $60 \mathrm{kA}$ \\
Inductance $L$ & $80 \mathrm{mH}$ \\
Operational Temperature $T_{\text {coil }}$ & $20 \mathrm{~K}$ \\
Series Joint Resistance ${ }^{a} R_{j}$ & $100 \mathrm{n} \Omega$ \\
Charging Time $t_{c h}$ & 24 hours \\
\hline \hline${ }^{*}$ Compact tokamak with 18 TF magnets with total inductance of 1.5 H. \\
${ }^{a}$ Resistance of HTS-HTS solder joints [34].
\end{tabular}

does not become saturated [28]. Common methods of joining HTS coated conductors introduce finite joint resistances, and will negatively impact the efficiency of the device. Inductance in the charging loop will introduce finite loss during transitions between the charging and maintenance phases of the input waveform. Including these terms is beyond the scope of the present work.

\section{CRyogenic Heat LoAd}

The ideal efficiency model presented in Sec. II may be used to calculate the expected heat load of switching in the cryogenic environment. From (2), the switching loss can be calculated from a given power output $P_{\text {out }}$ and efficiency.

$$
P_{s w}=\dot{Q}=P_{\text {out }}\left(\frac{1}{\eta}-1\right)
$$

where $\dot{Q}$ is the cryogenic heat load of the flux pump. To calculate the cryogenic heat load of an ideal TRFP flux pump, one need only know the power output and efficiency, determined by switching factor $\kappa$.

\section{Design Parameters of a 60 KA Flux Pump}

Using the models presented in Sec. II and III, the design parameters of a $60 \mathrm{kA}$ Flux Pump for a fusion TF coil can be determined. The load considered is a reactor-like magnet, whose specifications are summarized in Table I. To achieve the rated current capacity, the flux pump is constructed from cable containing a number of parallel tapes, where the number of tapes can be calculated with (11) as $i_{L} / I_{c, b}\left(B_{a, \perp}\right)(\kappa-1)$. Decreasing the operational temperature of the flux pump increases the self-field critical current, reducing the number of parallel tapes needed. However, it also reduces critical current suppression due to applied field, reducing switching factor. The operational temperature can then be optimized to minimize the size of cable required. In the proceeding example, an operational temperature of $60 \mathrm{~K}$ and $1.0 \mathrm{~T}$ of applied field are chosen. This could be achieved by locating the flux pump in a separate cryostat. The resulting design parameters of a $60 \mathrm{kA}$ flux pump are summarized in Table II.

The cryogenic heat load from switching can be calculated from required output power and device efficiency using (17). During maintenance mode, the flux pump need only overcome the joint resistance of the magnet, expected to be on the order of $100 \mathrm{n} \Omega$ [34], resulting in an output power of $360 \mathrm{~W}$. An average output of $55 \mathrm{mV}$ is required to reach $60 \mathrm{kA}$ in a 24-hour charging period. This results in average and maximum power outputs of $1.65 \mathrm{~kW}$ and $3.3 \mathrm{~kW}$, respectively.
TABLE II

Design Parameters of a 60 KA Flux PumP

\begin{tabular}{ll}
\hline \hline Switch Temperature $T_{s w}$ & $60 \mathrm{~K}$ \\
Switch Applied Field $B_{a, \perp}$ & $1.0 \mathrm{~T}$ \\
\hline Switching Factor $\kappa$ & 6.4 \\
Full-wave Efficiency $\eta$ & $84 \%$ \\
Parallel Tapes $n$ & 45 \\
\hline Charging Voltage $V_{D C}$ & $55 \mathrm{mV}$ \\
Charging Power $P_{c h}($ avg./max.) & $1650 / 3300 \mathrm{~W}$ \\
Heat Load $\dot{Q}_{c h}(\eta=84 \%)$ & $314 / 728 \mathrm{~W}$ \\
\hline Maintenance Voltage $V_{\text {pers }}$ & $6 \mathrm{mV}$ \\
Maintenance Power $P_{\text {pers }}$ & $360 \mathrm{~W}$ \\
Heat Load $\dot{Q}_{\text {pers }}(\eta=84 \%)$ & $69 \mathrm{~W}$ \\
\hline \hline
\end{tabular}

Such voltage outputs are achievable with HTS switching elements [35]. For a full-wave flux pump under the chosen operating parameters, the resulting cryogenic heat loads during charging and maintenance modes are then $314 \mathrm{~W}$ and $69 \mathrm{~W}$, respectively. ITER plans to use binary copper-HTS current leads [5], with an intermediate stage at $65 \mathrm{~K}$. The copper stage optimally leaks about $25 \mathrm{~W} / \mathrm{kA}$ to this intermediate stage [6]. A pair of current leads carrying $60 \mathrm{kA}$ would leak $3 \mathrm{~kW}$ of heat to the intermediate stage. This load could be directly replaced by a flux pump solution, providing an approximately 90\% reduction in cryogenic load during charging, and $98 \%$ reduction while maintaining the load at $60 \mathrm{kA}$.

The overall size of the flux pump can also be estimated. The main components of the flux pump are the transformer and switches. The cross-sectional area to prevent the transformer from saturating is well understood, and can be calculated from

$$
S_{\text {iron }}=\frac{V_{D C}}{B_{\text {sat }} f N_{2}}
$$

where $V_{D C}$ is the dc output voltage, $N_{2}$ is the number of turns of the superconducting secondary, $\beta$ is the saturation field of the iron core, and $f$ is the frequency of operation. This is larger than what is required by an ac sine wave input to allow for the derating of the transformer [36]. For a flux pump operating at $10 \mathrm{~Hz}$ with $N_{2}=10$, a core area of $1225 \mathrm{~mm}^{2}$ will be enough to supply $110 \mathrm{mV}$. The room temperature copper windings will not contribute significantly to the size of the transformer. The transformer volume can be estimated to be only $0.008 \mathrm{~m}^{3}$. The switch can similarly be calculated to have a volume of $0.079 \mathrm{~m}^{3}$, or $0.16 \mathrm{~m}^{3}$ for a pair. The total volume of the flux pump is expected to be $<0.2 \mathrm{~m}^{3}$. Therefore, an reactor-like tokamak with 18 field coils would need a flux pump cryostat of only $3.6 \mathrm{~m}^{3}$; comparable to a standard server rack. This may be separate from the reactor cryostat, linked by HTS current leads [5]. Additional room temperature components include the transformer and switch coil power supplies, which can be of modest size. Such a flux pump implementation is expected to be much smaller than the 5 ha. of switching and bus bars implemented for the ITER project [4], reducing the capital and maintenance costs of the TF current supply system.

\section{CONCLUSION}

In this work, we have presented an ideal power transfer efficiency model for transformer-rectifier flux pumps. In this model, efficiency depends only on the switch performance, 
represented by the switching factor $\kappa$. Switch performance and subsequent efficiency is improved by increasing applied field and operating at temperatures with favourable $J_{c}(B)$ characteristics. Efficiencies of $>80 \%$ can be achieved by a flux pump operating at $60 \mathrm{~K}$ with only $1.0 \mathrm{~T}$ of applied field in both the half- and full-wave circuit topologies.

The ideal efficiency model presented was used to calculate the expected heat load of a transformer-rectifier flux pump when charging and maintaining the coil current of a reactorlike TF magnet. It was found that for a full-wave flux pump operating at $60 \mathrm{~K}$ and $1.0 \mathrm{~T}$ of applied field, the expected heat load during charging and current maintenance is only $314 \mathrm{~W}$ and $69 \mathrm{~W}$ respectively. This is almost 100 times less than the heat leak associated with gas-cooled copper current leads to room temperature. Such a flux pump solution would reduce the cryogenic cooling power required, and thus improve the overall power balance of a tokamak reactor. Flux pumping also enables the toroidal field to be sustained persistently, allowing for steady-state reactor designs.

The presented model is idealized and simplistic to ensure initial tractability. It is expected that transformer and switch coil losses, finite circuit inductance, joint resistance, and ac loss in the circuit will all contribute to the overall wall-plug efficiency of a transformer rectifier flux pump. Future work will be needed to address these issues.

\section{REFERENCES}

[1] N. Mitchell, D. Bessette, R. Gallix, C. Jong, J. Knaster, P. Libeyre, C. Sborchia, and F. Simon, "The ITER Magnet System," IEEE Trans. Appl. Supercond., vol. 18, no. 2, pp. 435-440, Jun. 2008.

[2] H. W. Weijers et al., "High Field Magnets With HTS Conductors," IEEE Trans. Appl. Supercond., vol. 20, no. 3, pp. 576-582, Jun. 2010.

[3] R. Aymar, P. Barabaschi, and Y. Shimomura, "The ITER design," Plasma Phys. Control. Fusion, vol. 44, no. 5, pp. 519-565, Apr. 2002.

[4] J. Tao, I. Benfatto, J. K. Goff, A. Mankani, F. Milani, I. Song, H. Tan, and J. Thomsen, "ITER Coil Power Supply and Distribution System," in 2011 IEEE/NPSS 24th Symposium on Fusion Engineering, Jun. 2011.

[5] A. Ballarino et al., "Design of the HTS Current Leads for ITER," IEEE Trans. Appl. Supercond., vol. 22, no. 3, p. 4800304, Jun. 2012.

[6] A. Ballarino, "Current Leads, Links and Buses," Proc. of the CASCERN Accelerator School: Superconductivity for Accelerators (Erice, Italy), 2013

[7] V. Kalinin, E. Tada, F. Millet, and N. Shatil, "ITER cryogenic system," Fusion Engineering and Design, vol. 81, no. 23, pp. 2589-2595, Nov. 2006.

[8] L. van de Klundert and H. ten Kate, "Fully superconducting rectifiers and fluxpumps Part 1: Realized methods for pumping flux," Cryogenics, vol. 21, no. 4, pp. 195-206, Apr. 1981.

[9] L. J. M. van de Klundert and H. H. J. ten Kate, "On fully superconducting rectifiers and fluxpumps. A review. Part 2: Commutation modes, characteristics and switches," Cryogenics, vol. 21, no. 5, pp. 267-277, May 1981.

[10] M. P. Oomen, M. Leghissa, G. Ries, N. Proelss, H. Neumueller, F. Steinmeyer, M. Vester, and F. Davies, "HTS flux pump for cryogen-free HTS magnets," IEEE Trans. Appl. Supercond., vol. 15, no. 2, pp. 1465-1468, Jun. 2005.

[11] Z. Bai, G. Yan, C. Wu, S. Ding, and C. Chen, "A novel high temperature superconducting magnetic flux pump for MRI magnets," Cryogenics, vol. 50, no. 10, pp. 688-692, Oct. 2010.

[12] C. Hoffmann, D. Pooke, and A. D. Caplin, "Flux Pump for HTS Magnets," IEEE Trans. Appl. Supercond., vol. 21, no. 3, pp. 1628-1631, Jun. 2011.

[13] J. Geng and T. A. Coombs, "Mechanism of a high-Tc superconducting flux pump: Using alternating magnetic field to trigger flux flow," Appl. Phys. Lett., vol. 107, no. 14, p. 142601, Oct. 2015.
[14] J. Geng and T. A. Coombs, "An HTS flux pump operated by directly driving a superconductor into flux flow region in the E-J curve," Supercond. Sci. Technol., vol. 29, no. 9, p. 095004, Jul. 2016.

[15] T. A. Coombs, J. Geng, L. Fu, and K. Matsuda, "An Overview of Flux Pumps for HTS Coils," IEEE Trans. Appl. Supercond., vol. 27, no. 4 , p. 4600806, Jun. 2017.

[16] J. Ma, J. Geng, and T. A. Coombs, "Flux pumping for non-insulated and metal-insulated HTS coils," Supercond. Sci. Technol., vol. 31, no. 1, p. 015018 , Dec. 2017.

[17] P. Zhou, G. Ma, Y. Deng, X. Nie, Y. Zhai, K. Liu, H. Zhang, and Y. Li, "A Contactless Self-Regulating HTS Flux Pump," IEEE Trans. Appl. Supercond., vol. 30, no. 4, p. 3603006, Jun. 2020.

[18] R. Mataira, M. Ainslie, A. Pantoja, R. Badcock, and C. Bumby, "Mechanism of the High-Tc Superconducting Dynamo: Models and Experiment," Phys. Rev. Applied, vol. 14, no. 2, p. 024012, Aug. 2020.

[19] K. Hamilton, A. E. Pantoja, J. G. Storey, Z. Jiang, R. A. Badcock, and C. W. Bumby, "Design and Performance of a "Squirrel-Cage" Dynamo-Type HTS Flux Pump," IEEE Trans. Appl. Supercond., vol. 28, no. 4, p. 5205705, Jun. 2018.

[20] J. Geng, T. Painter, P. Long, J. Gawith, J. Yang, J. Ma, Q. Dong, B. Shen, C. Li, and T. A. Coombs, "A kilo-ampere level HTS flux pump," Supercond. Sci. Technol., vol. 32, no. 7, p. 074004, Jul. 2019.

[21] J. Geng, C. W. Bumby, and R. A. Badcock, "Maximising the current output from a self-switching kA-class rectifier flux pump," Supercond. Sci. Technol., vol. 33, no. 4, p. 045005, Apr. 2020.

[22] S. Lee et al., "Persistent Current Mode Operation of A 2G HTS Coil With A Flux Pump," IEEE Trans. Appl. Supercond., vol. 26, no. 4, p. 0606104, Jun. 2016.

[23] Z. Jiang, K. Hamilton, N. Amemiya, R. A. Badcock, and C. W. Bumby, "Dynamic resistance of a high-Tc superconducting flux pump," Appl. Phys. Lett., vol. 105, no. 11, p. 112601, Sep. 2014.

[24] C. W. Bumby, A. E. Pantoja, H. Sung, Z. Jiang, R. Kulkarni, and R. A. Badcock, "Through-Wall Excitation of a Magnet Coil by an External-Rotor HTS Flux Pump," IEEE Trans. Appl. Supercond., vol. 26, no. 4, p. 0500505, Jun. 2016.

[25] J. D. D. Gawith, J. Geng, C. Li, B. Shen, X. Zhang, J. Ma, and T. A. Coombs, "A half-bridge HTS transformer-rectifier flux pump with two AC field-controlled switches," Supercond. Sci. Technol., vol. 31, no. 8, p. 085002 , Jun. 2018.

[26] R. C. Mataira, M. D. Ainslie, R. A. Badcock, and C. W. Bumby, "Origin of the DC output voltage from a high-Tc superconducting dynamo," Appl. Phys. Lett., vol. 114, no. 16, p. 162601, Apr. 2019.

[27] B. Leuw, J. Geng, J. H. P. Rice, D. Moseley, and R. A. Badcock, "Demonstration of a half-wave superconducting transformer-rectifier flux pump (strfp) using Jc(B) switches," 2021, submitted manuscript.

[28] C. Li, S. Wang, H. Jia, J. He, B. Li, and T. A. Coombs, "Impacts of the Saturated Transformer on the HTS Flux Pump," IEEE Trans. Appl. Supercond., vol. 31, no. 8, p. 5500504, Nov. 2021.

[29] K. Hamilton, R. Mataira, J. Geng, C. Bumby, D. Carnegie, and R. Badcock, "Practical Estimation of HTS Dynamo Losses," IEEE Trans. Appl. Supercond., vol. 30, no. 4, p. 4703105, Jun. 2020.

[30] C. P. Bean, "Magnetization of High-Field Superconductors," Rev. Mod. Phys., vol. 36, no. 1, pp. 31-39, Jan. 1964.

[31] N. M. Strickland, C. Hoffmann, and S. C. Wimbush, "A 1 kA-class cryogen-free critical current characterization system for superconducting coated conductors," Review of Scientific Instruments, vol. 85, no. 11, p. 113907, Nov. 2014.

[32] S. C. Wimbush and N. M. Strickland, "A Public Database of High-Temperature Superconductor Critical Current Data," IEEE Trans. Appl. Supercond., vol. 27, no. 4, p. 8000105, Jun. 2017.

[33] A. Ghabeli, M. Ainslie, E. Pardo, L. Queval, and R. Mataira, "Modeling the charging process of a coil by an HTS dynamo-type flux pump," Supercond. Sci. Technol., vol. 34, no. 8, p. 084002, Jul. 2021.

[34] Y. Kim, J. Bascunan, T. Lecrevisse, S. Hahn, J. Voccio, D. K. Park, and Y. Iwasa, "YBCO and Bi2223 Coils for High Field LTS/HTS NMR Magnets: HTS-HTS Joint Resistivity," IEEE Trans. Appl. Supercond., vol. 23, no. 3, p. 6800704, Jun. 2013.

[35] C. Li, J. Geng, J. Gawith, B. Shen, X. Zhang, H. Zhang, J. Ma, and T. A. Coombs, "Design for a Persistent Current Switch Controlled by Alternating Current Magnetic Field," IEEE Trans. Appl. Supercond., vol. 28, no. 4, p. 4603205, Jun. 2018.

[36] C. P. Steinmetz, "On the law of hysteresis," AIEE Transactions, vol. 9, pp. 3-64, 1892. 